\title{
Costs of a clinical chemistry laboratory
}

\author{
JA STILWELL \\ From the Health Service Research Centre, University of Birmingham, Edgbaston, Birmingham B15 2TJ
}

SUMMARY The costs of a clinical chemistry laboratory in a district general hospital were studied. The system used has certain advantages over the conventional Cooper Lybrand method. The time taken by technicians to perform tests was more variable than expected and the cost of sample collection was higher than process-cost for many tests. Indirect costs (overheads) were greater than direct costs and there were potential economies of scale. The most time-consuming part of this study was collecting the cost of chemicals and other disposables.

The Health Services Research Centre of the University of Birmingham is studying methods of evaluating the use of diagnostic tests in hospitals. Part of the work has been a study of the costs of a clinical chemistry laboratory in a district general hospital between 1977 and 1978, and this paper presents the method used, and the resulting costs. The technique recommended by the Department of Health for laboratory costing studies was developed by Cooper and Lybrand and Associates Ltd. ${ }^{1}$ We considered that this approach has serious theoretical and practical drawbacks and it was not, therefore, adopted.

The Cooper Lybrand method of laboratory cost analysis is open to a number of criticisms:

(i) the method involves considerable cost in staff time;

(ii) it does not offer any theoretical justification or explanation for the procedures it recommends;

(iii) the cost of collecting samples is not accurately attributed for grouped tests;

(iv) the recommended estimation of process labour costs is inadequate because of the variability of the length of process times;

(v) Cooper Lybrand impute no interest to capital employed and write the equipment off in 7 years. This leads to valuation errors.

(vi) the report does not cover the whole range of workers in the laboratory-for example, cleaners;

(vii) no guidance is given on the treatment of direct or indirect taxes;

(viii) the cost of energy and other utilities is not included.

The clinical chemistry laboratory in this study serves a metropolitan health district. It also provides a service to the outpatient department (about $14 \%$ of

Accepted for publication 13 October 1980 work load) and to general practitioners (about $7 \cdot 5 \%$ of work load).

At the time of the study the laboratory was producing annually, some 98500 test results, of which 42500 were profiles consisting of 10 elements. Counting each element of the profile as a separate test, the annual production was approximately 481000 . The laboratory employed 19 full-timeequivalent professional staff. A Wang 2200 computer was used for data processing, with an autoanalyser chart reader. Small batch results and patient identities were entered manually.

\section{Definitions and economic assumptions}

Direct costs are the costs of the inputs essential and exclusive to a particular determination at a particular time. They exclude therefore, such costs as the cost of lighting or supervision, but they include the cost of the equipment employed.

Indirect costs are total costs minus direct costs. Supervision and laboratory administration are the largest element of indirect costs. Indirect costs were allocated to tests according to the level of direct costs, thus preserving cost relativities.

Marginal costs of a particular test are the extra costs of performing one additional test of the same type. Average total costs of a test are the total costs of the resources devoted to the test, divided by the number of test results produced.

Average direct cost - This is average total cost minus average indirect cost.

Capital-The capital of the laboratory is defined as those pieces of equipment not subject to the routine ordering procedure used to purchase reagents, disposables, etc.

Capital cost-There are two elements of capital cost. ${ }^{2}$ In the first place, the allocation of resources to 
laboratory equipment means that these resources are not available for other purposes. In the second place the equipment has a finite life. The appropriate method to take account of these factors is to calculate the mortgage formula:

Principal $\times$ rate of interest expressed as decimal $1-(1+\text { rate of interest })^{-n}$

to this should be added the cost of annual maintenance.

Capital cost assumptions - In this study an estimated equipment life of 12.5 years was used. Some of the equipment was older than this and in value terms, a large proportion was younger and of a recent technical vintage. The estimated life of this equipment had to be an educated guess. The rate of interest used was the Government Test Discount rate of $7 \%$ in real terms. All capital costs except for the amortisation of the data processing computer and equipment such as typewriters used by staff who counted as indirect labour were allocated to tests as direct costs. Indirect capital costs were imputed to tests according to relative direct total costs.

Materials are defined as all the items purchased by means of the routine ordering procedure-reagents, pipettes, analyser cups, kits, etc.

Materials cost assumption - The cost figures used were prices paid minus VAT (value added tax). ${ }^{3}$

Labour input to the laboratory is everyone who works there, or in the hospital collecting samples or distributing results. This includes secretaries, cleaners, porters, biochemists, and medical practitioners as well as technicians and phlebotomists.

Labour cost assumption-The cost figures used were based upon gross wages, plus National Insurance and Superannuation. Staff engaged in research and teaching were included and their labour was entered as an indirect cost.

\section{Method of costing individual tests}

The direct cost of a test falls naturally into two parts; the collection of the sample and its preparation for laboratory procedure, and the performance of the procedure and transmission of results. Labour, materials and capital are used in each part.

\section{Labour, direct costs}

(a) Collection costs-At the hospitals which use the laboratory under study, a mixed system of blood collection and preparation was employed. Eleven 13-hour per week phlebotomists were employed, other samples being collected by laboratory technicians, ward staff and GPs. The cost of collecting a sample was taken as the estimated phlebotomist cost.

A survey was undertaken over 14 days in order to allocate the work of the phlebotomists to the separate laboratories. The same survey was used within the clinical chemistry laboratory to group tests according to the number of times they were requested in combination, and therefore gave rise to a split sample. A "collection factor" was estimated for each test, such that a test always carried out individually scored 1 , a test carried out with a mean of one other test scored 0.5 , a test carried out with a mean of two other tests scored $0.33 \mathrm{etc}$. The collection cost of each test therefore $=$ (total phlebotomist wages $)$ $\div$ (total number of samples taken) $\times$ (allocation factor to clinical chemistry laboratory) $\times$ (collection factor). Samples other than blood required a separate ad hoc estimate of collection cost.

(b) Process costs-A technician-cost per minute was calculated from the mid-point of the MLSO scale. A time-accumulation clock was placed by the equipment used for each test and this was switched on by the technician when performing an activity associated with the test starting and ending with the necessary clerical work and encompassing all the process activities. The clock would be switched off if the technician left the test during its process time, to do something else. The accumulated time was therefore technician-involvement time, not machine-running time.

Between eight and twelve observation were obtained for most tests. It was not practicable to use this method for some tests which were performed infrequently, and for these the technicians used informally, the same self-recording technique as that recommended by Cooper Lybrand. ${ }^{1}$

\section{Materials, direct cost}

(a) Collection-The expensive items were syringes, needles, and sterettes. Their costs were allocated to tests according to the allocation and collection factors described above.

(b) Process-In order to assess and cost the use of materials, a questionnaire was issued for each test to the technician responsible for that test. This identified for each test: (i) disposable items and their frequencies of use; (ii) the constituents of reagents and their utilisation; (iii) control sera employed; (iv) calibration material employed. The cost per test was then calculated from this information, and entered as the direct process cost.

\section{Capital, direct costs}

(a) Collection-Current replacement costs were found for the centrifuges, refrigerators, and freezer. The annual cost was calculated according to the formula given above, and allocated in the same way as the labour direct costs.

(b) Process-Current replacement costs were found 
for all items of equipment. These were allocated, where the equipment was shared, according to the relative time used by each test type. A worked example (the calculation of the cost of the Gamma Counter) is available upon application.

\section{Labour indirect costs}

These were estimated very simply by subtracting the sum of annual estimated direct costs from the actual annual labour bill.

\section{Materials, indirect costs}

The same procedure was used as for indirect labour costs.

\section{Capital, indirect costs}

The annual costs of the data processing equipment were allocated equally between all tests.

\section{Space overheads}

This is the cost of heat, water, and electricity used for light. The method of making the estimate was very simple; the annual energy bill was multiplied by the factor $\mathrm{m}^{3}$ (laboratory) $/ \mathrm{m}^{3}$ (whole hospital). The numerator was measured, the denominator is a figure held by the hospital engineer's department.

\section{Results}

The total cost of the 98500 results was $£ 190000$. The overall average cost per test excluding profiles, was $£ 1.94$. The average cost of a profile was $£ 1.91$. Laboratory resources are concentrated on the

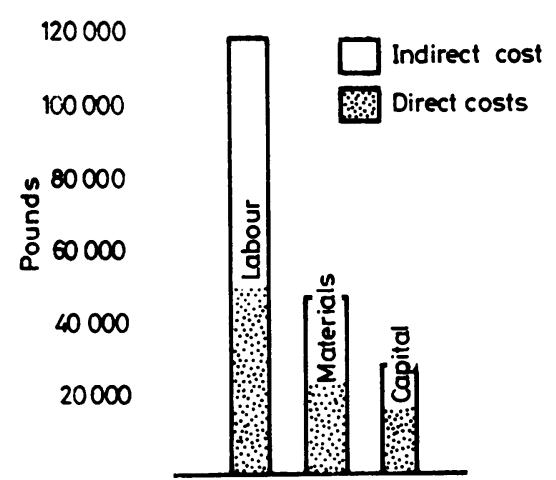

Fig. 1 Annual cost of a chemical pathology laboratory.

production of relatively few tests. Excluding profiles, 14 tests out of a total of about 60 different tests account for $75 \%$ of laboratory resources, and $50 \%$ of resources are accounted for by eight tests. Fig. 1 shows labour, materials and capital cost divided into direct and indirect cost.

Figures for all tests are available upon application.

\section{Labour, direct costs}

In the Table, (a) shows the estimated average direct labour costs for a sample of tests performed during the survey period, separated into collection cost and process cost (figures for all tests are available upon application). Total direct labour cost was estimated at $£ 46000$.

Elements of cost-selected tests (pence per test)

\begin{tabular}{|c|c|c|c|c|c|c|c|}
\hline & $\begin{array}{l}\text { Estimate of } \\
\text { annual no. } \\
\text { performed }\end{array}$ & $\begin{array}{l}\text { Collection } \\
\text { cost }\end{array}$ & $\begin{array}{l}\text { Process } \\
\text { cost }\end{array}$ & $\begin{array}{l}\text { Direct } \\
\text { cost }\end{array}$ & $\begin{array}{l}\text { Indirect } \\
\text { cost }\end{array}$ & $\begin{array}{l}\text { Marginal } \\
\text { cost }\end{array}$ & Total cost \\
\hline \multicolumn{8}{|l|}{ (a) Labour costs } \\
\hline Profile & 42500 & 19 & 26 & 45 & 78 & 22 & 123 \\
\hline Glucose & 9500 & 10 & 21 & 31 & 32 & 31 & 63 \\
\hline T3 uptake & 4650 & 10 & 13 & 23 & 24 & 13 & 47 \\
\hline Acid phosphatase & 1600 & 17 & 24 & 41 & 42 & 29 & 83 \\
\hline $\begin{array}{l}\text { Electrophoretic strip } \\
\text { (b) Materials cost }\end{array}$ & 550 & 17 & 27 & 44 & 46 & 21 & 90 \\
\hline Profile & & 8 & 17 & 25 & 23 & 25 & 48 \\
\hline Glucose & & 4 & 15 & 19 & 19 & 19 & 38 \\
\hline T3 uptake & & 4 & 16 & 20 & 20 & 20 & 40 \\
\hline Acid phosphatase & & 7 & 2 & 9 & 9 & 9 & 18 \\
\hline Electrophoretic strip & & 7 & $\overline{9}$ & 16 & 16 & 16 & 32 \\
\hline $\begin{array}{l}\text { Profile } \\
\text { Prit }\end{array}$ & & & & 11 & 9 & & 20 \\
\hline Glucose & & & & 6 & 9 & & 15 \\
\hline T3 uptake & & & & 13 & 9 & & 22 \\
\hline Acid phosphatase & & & & 27 & 9 & & 36 \\
\hline $\begin{array}{l}\text { Electrophoretic strip } \\
\text { (d) Overall }\end{array}$ & & & & - & 9 & & 9 \\
\hline Profile & & 27 & 43 & 81 & 110 & 47 & 191 \\
\hline Glucose & & 14 & 36 & 56 & 60 & 50 & 116 \\
\hline T3 uptake & & 14 & 29 & 56 & 53 & 33 & 109 \\
\hline Acid phosphatase & & 24 & 26 & 77 & 60 & 38 & 137 \\
\hline Electrophoretic strip & & 24 & 36 & 60 & 71 & 37 & 131 \\
\hline
\end{tabular}


Materials, direct costs

In the Table, (b) shows the estimated direct materials cost for the tests performed during the survey period, separated into collection cost and process cost.

\section{Capital, direct costs}

Total annual direct capital cost was estimated at $£ 17$ 500. Table (c) shows the estimated direct capital cost per test.

\section{Labour, indirect costs}

Indirect labour cost was $£ 75500$. About two thirds of indirect labour costs were the salaries of director, chief technician, secretary and clerk, porters, washers, and cleaners and most of the salaries of the biochemists, amounting to approximately $£ 42000$. The remaining labour costs represent slack periods between tests, routine procedure which apply to more than one test, or training etc. Column 5 of the Table shows apportioned indirect labour costs.

\section{Materials, indirect costs}

This was estimated at $£ 21000$. Column 5 of the Table shows indirect costs apportioned to tests.

\section{Capital, indirect costs and space overheads}

These were estimated at $£ 3300$ and $£ 4750$, yielding $9 \mathrm{p}$ per test.

\section{Average direct costs and marginal costs}

Marginal costs are below direct costs because the latter include fixed equipment and because typically, a test run involves a set-up time which is independent of the number of individual tests processed. Figs. 2-4 show the observations on three tests. By fitting a line to the observations it is possible to divide lapsed time into an element (equal to the vertical intercept) corresponding to set-up time, and an element corresponding to marginal time. For example, it appears from Fig. 2 that the time taken to process T3 uptake tests can be divided into two components-a

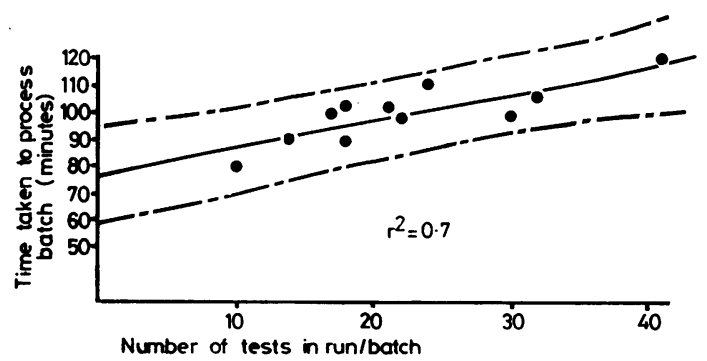

Fig. 2 The relation between pr cess time and run length (T3 uptake).

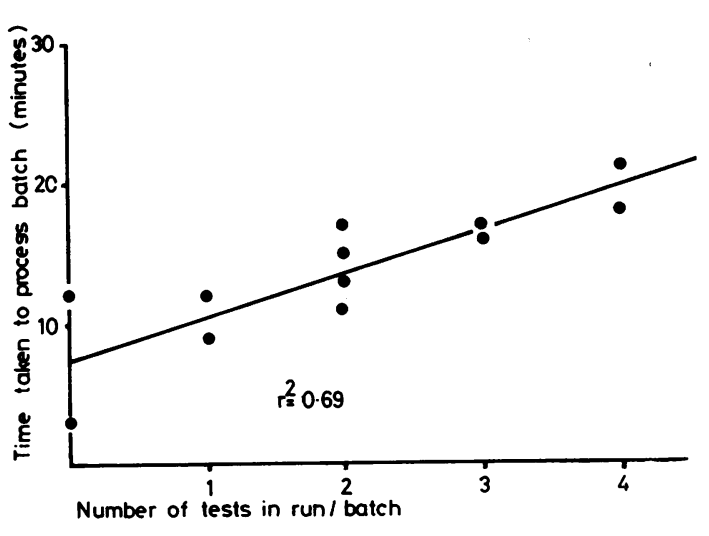

Fig. 3 The relation between process time and run length (thymol turbidity).

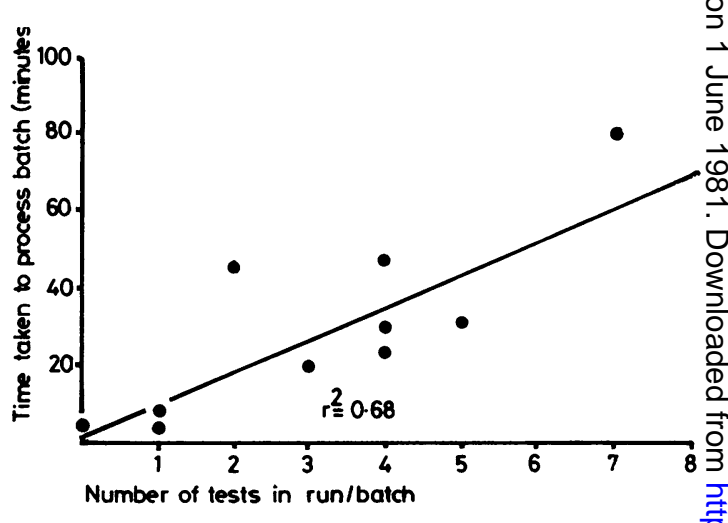

Fig. 4 The relation between process time and run length (amylase).

set-up time which is independent of the number of tests in the run, and a process time which bears an linear relationship (as would be expected) to the number of tests. The estimate of the constant time component is 77 minutes, and the process time is estimated at one minute per test.

The sample of observations upon T3 uptake gave the best statistical fit; the majority of sampleser yielded a considerably lower correlation. Fig. 5, forn example, shows the scatter of observations upon sodium and potassium, when not done as part of a profile. In this case it was necessary to derive the best estimate from interview. Marginal costs are shown in column 6 of the Table.

Although therefore, for the majority of tests marginal costs were well below direct costs, for abouts a quarter of all tests direct cost and marginal cost were roughly the same. The mean relation between marginal and direct cost was that marginal $\cos \Omega$ equalled $68 \%$ of direct cost. 


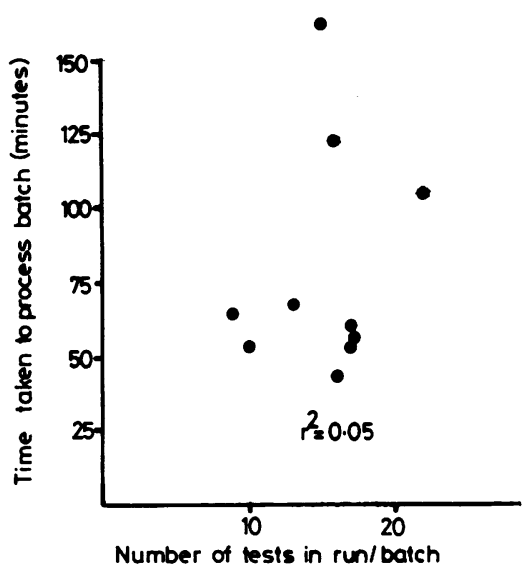

Fig. 5 The relation between process time and run length (sodium and potassium).

\section{Capital/labour ratios}

Some tests use more capital relative to labour than others, but the relative insignificance of capital cost can be seen from the fact that for only six separate tests, accounting for some $17 \%$ of through-put, counting a profile as one test (or for some $3.5 \%$ of through-put counting a profile as ten tests), is the capital/labour ratio greater than 1 . For $60 \%$ of through-put the capital/labour ratio is under $\mathbf{0} \cdot 2$ such tests using more than five times as much labour as capital.

\section{Cost distribution between tests}

Fig. 6 shows the distribution of average total cost per test (excluding profiles which cost $£ 1.91$ each). As can easily be seen, the limits $75 p$ to $£ 2.00$ encompass $75 \%$ of tests performed. Some of the tests are expensive because thay take up a great deal of technician time-for example, xylose tolerance, others because expensive reagents are required-for example, immunoglobulins and some because they employ expensive equipment which is not used to capacity-for example, chloride. A worked example is available upon application.

\section{Discussion}

Many studies have examined the cost of particular determinations or have looked at laboratory costs with a view to an evaluation of the use of some particular equipment, ${ }^{4-6}$ but the main purpose of this paper is to present a cross-sectional cost picture of a clinical laboratory in a busy general hospital. Some points to note are the heavy weight of indirect costs, the divergence between average and marginal costs

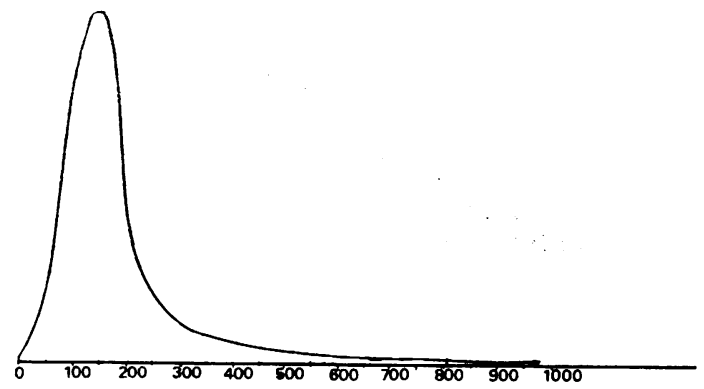

Fig. 6 Relative distribution of test average direct costs excluding profile (pence).

and the concentration of resources on the production of a relatively small set of tests.

After allowing for multiple requests from the same samples the average collection cost was $23 \mathrm{p}$. Collection labour cost is greater than marginal process technician cost in $75 \%$ of tests, and greater even than average process technician cost in $15 \%$ of tests. Profiles and isotope tests are produced at a low capital cost because of the high through-put; capital costs are in any case the lowest cost element, being only slightly higher than the phlebotomy costs, and we can conclude that, although utilising some very modern equipment, clinical chemistry is, as organised at present, still very labour intensive.

It is also clear, both from the existing level of equipment utilisation and more importantly from the observed overall shortfall of marginal below average cost, that substantial economies of scale could potentially be exploited. A secondary purpose of the paper is to present a costing method which is both more comprehensive and simpler to use than the method most commonly employed. Probably the most timeconsuming part of the costing exercise was deriving the cost of reagents, standards and controls. The use of time-accumulation clocks enabled reasonablysized samples to be taken of technician process-time, without on the one hand incurring the expense and intrusiveness of work study staff, or on the other presenting the technicians with the prolonged inconvenience of completing detailed time sheets.

I thank Mr Peter Scott, head of Biochemistry Department, Selly Oak Hospital, and Mr George Cummings, Senior Chief MLSO, for their advice and help.

\section{References}

1 Cooper and Lybrand and Associates Ltd. Procedure for determining test costs in pathology laboratories. London: Department of Health and Social Security, 1976.

2 Forbes JF. Capital valuation and the health services. Health Economics Research Unit, University of Aberdeen, 1979. 
${ }^{3}$ Layard R. In: Layard R, ed. Cost benefit analysis. London: Penguin, 1972.

${ }^{4}$ Buckley-Sharp MD, et al. Introduction of a Vickers M300 analyser into the routine service of a hospital laboratory. 1. Installation, staffing, logistics. J Clin Pathol 1976;29: 322-7.

${ }^{5}$ Richardson RW. Comparison of cost of preparing reagents in laboratory with cost of using commercial kits. Lancet 1977 ;ii:1273-5.
6 Rollason JG. Comprehensive costings for Technicon SMA 12/60 and SMA 6/60 systems: a three-year review. Med Lab Sci $1978 ; 35: 187-93$.

Requests for reprints to: Mr JA Stilwell, Health Service Research Centre, University of Birmingham, Edgbaston, Birmingham B15 2TJ, England. 